\title{
OPTIMAL DIVIDEND PAYMENTS UNTIL RUIN OF DIFFUSION PROCESSES WHEN PAYMENTS ARE SUBJECT TO BOTH FIXED AND PROPORTIONAL COSTS
}

\author{
JOSTEIN PAULSEN, ${ }^{*}$ University of Bergen
}

\begin{abstract}
The problem of optimal dividends paid until absorbtion at zero is considered for a rather general diffusion model. With each dividend payment there is a proportional cost and a fixed cost. It is shown that there can be essentially three different solutions depending on the model parameters and the costs. (i) Whenever assets reach a barrier $y^{*}$, they are reduced to $y^{*}-\delta^{*}$ through a dividend payment, and the process continues. (ii) Whenever assets reach a barrier $y^{*}$, everything is paid out as dividends and the process terminates. (iii) There is no optimal policy, but the value function is approximated by policies of one of the two above forms for increasing barriers. A method to numerically find the optimal policy (if it exists) is presented and numerical examples are given.
\end{abstract}

Keywords: Optimal dividend; diffusion model; impulse control; barrier strategy

2000 Mathematics Subject Classification: Primary 93E20

Secondary 49J15; 49K15; 60J70

\section{Introduction and model formulation}

In this paper the classical problem of maximizing the discounted value of dividends paid out from an income process is addressed. To get the mathematical formalism right, let $\left(\Omega, \mathcal{F},\left(\mathcal{F}_{t}\right)_{t \geq 0}, \mathrm{P}\right)$ be a probability space satisfying the usual conditions, i.e. the filtration $\left(\mathcal{F}_{t}\right)_{t \geq 0}$ is right continuous and P-complete. In this setup the income process without dividends is assumed to follow the dynamics

$$
\mathrm{d} X_{t}=\mu\left(X_{t}\right) \mathrm{d} t+\sigma\left(X_{t}\right) \mathrm{d} W_{t},
$$

where $W$ is a Brownian motion on the probability space and $\mu$ and $\sigma$ are functions defined on $[0, \infty)$.

From this income process dividends can be paid out to the owners, but at a cost. Total dividends paid out until time $t$ is given by $D_{t}$ and associated costs are given by $\bar{D}_{t}$. If the processes $D$ and $\bar{D}$ are nondecreasing, adapted, and right continuous with left limits (RCLL) then we say they are admissible.

The capital $Y$ retained in the company then has the dynamics

$$
\mathrm{d} Y_{t}=\mu\left(Y_{t}\right) \mathrm{d} t+\sigma\left(Y_{t}\right) \mathrm{d} W_{t}-\mathrm{d} D_{t}-\mathrm{d} \bar{D}_{t},
$$

with $Y_{0-}=y$. Time of ruin is defined as $\tau_{y}=\inf \left\{t: Y_{t}<0\right\}$ with $\tau_{y}=\infty$ if $Y_{t} \geq 0$ for all $t$. If $\mathscr{D}$ is the set of all admissible dividend policies with associated admissible costs, our task is

Received 13 March 2007; revision received 21 June 2007.

* Postal address: Department of Mathematics, University of Bergen, Johs. Brunsgt. 12, 5008 Bergen, Norway.

Email address: jostein@mi.uib.no 
to find

$$
V^{*}(y)=\sup _{D} \mathrm{E}^{y}\left[\int_{0-}^{\tau_{y}} \mathrm{e}^{-r t} \mathrm{~d} D_{t}\right],
$$

where $r$ is a properly chosen discount factor. Here, $\mathrm{E}^{y}$ is the expectation with $Y_{0-}=y$. Furthermore, if it exists, we also want to find the optimal policy $D^{*} \in \mathcal{D}$.

In this paper it is assumed that the cost process $\bar{D}$ consists of a fixed cost $d_{0}$ for each payment, independent of the size of the payment, plus a part that is proportional to the size of the payment. Of course, when there is a fixed cost for every dividend payment, there can only be a finite number of such payments on any finite time interval, since ruin will occur after a finite number of payments due to the cost with each payment. Therefore, we can write

$$
\bar{D}_{t}=d_{0} \sum_{s \leq t} \mathbf{1}_{\left\{\triangle D_{s}>0\right\}}+d_{1} D_{t},
$$

where $d_{1} \geq 0$ is the proportionality factor, $\mathbf{1}_{\{\cdot\}}$ denotes the indicator function, and $\triangle D_{s}=$ $D_{s}-D_{s-}$. Clearly, this $\bar{D}$ is admissible whenever $D$ is. From (1.1) and (1.2) it trivially follows that

$$
\begin{aligned}
\Delta Y_{t} & =-\left(1+d_{1}\right) \Delta D_{t}-d_{0} \mathbf{1}_{\left\{\triangle D_{t}>0\right\}}, \\
\Delta D_{t} & =\frac{\left(-\Delta Y_{t}\right)-d_{0} \mathbf{1}_{\left\{\triangle D_{t}>0\right\}}}{1+d_{1}} .
\end{aligned}
$$

The first of these equations gives the negative of the amount the assets are reduced with in order to pay $\triangle D_{t}$ in dividends, and the second gives the dividends received when assets are reduced with $\triangle Y_{t}$.

A more general cost function is in principle possible, but at the expense of a much more delicate analysis.

Under the assumptions given in Section 2, when $d_{0}=0$ this problem was solved by Shreve et al. (1984). They showed that the optimal dividend process, if it exists, is a singular process. We shall return to their solution in Section 2. The special case when $X$ is a Brownian motion with drift, i.e. when $\mu$ and $\sigma$ are constants, was dealt with in, for example, Asmussen and Taksar (1997), Gerber and Shiu (2004), and Jeanblanc-Picqué and Shiryaev (1995) (the latter also solved the case where $d_{0}>0$ ). In Cadenillas et al. (2007), $d_{0}$ is again positive and $X$ is a special Ornstein-Uhlenbeck process. Cadenillas et al. also allowed a more general cost function. When $d_{0}>0$, the optimal dividend process is a jump process, since dividends are paid in lumps whenever the capital reaches a barrier. This kind of solution is called an impulse control solution. Some other particular solutions when $d_{0}=0$ can be found in Paulsen and Gjessing (1997) and Cai et al. (2006). Lungu and Øksendal (1997) considered the problem of optimal harvesting of renewable resources until extinction, and they provided a solution for a diffusion growth model when $d_{0}=0$. Their model was not covered by the general model in Shreve et al. (1984), but again the optimal control is singular. In Paulsen (2003) the general model of Shreve et al. (1984) was analyzed in the context of solvency constraints on capital until dividends can be paid.

Alvarez (2004a) considered a rather general diffusion model with a more general cost function then (1.3). However, in his model $Y$ is reduced to a fixed $y_{0}$ whenever payments (or harvesting) are made. In Alvarez (2004b) this kind of problem was analyzed in the context of deciding optimal harvesting of timber. This is in fact a classical problem; see Alvarez (2004b) for references. 
A topic we shall not enter here is optimal dividend payments combined with optimal choice of the size of the business and riskiness of investments. References here are Højgaard and Taksar (2004) and Choulli et al. (2003), when $d_{0}=0$, and Cadenillas et al. (2006), when $d_{0}>0$. The models studied in these papers are linear Brownian motions subject to control of exposure and possibly also compounded with another linear Brownian motion. Different extensions can be found in Décamps and Villeneuve (2007) where dividend payments were combined with an optimal stopping problem to decide when investments should be done, and in Grandits et al. (2007) where the objective was to maximize expected exponential utility of dividends paid. Again, in both these papers $X$ is a Brownian motion with drift and $d_{0}=0$.

The purpose of this paper is to give a complete solution to the optimal dividend problem for the general model presented by Shreve et al. (1984) when $d_{0}>0$, thus extending the results of Jeanblanc-Picqué and Shiryaev (1995) and, with a less general cost function, those of Cadenillas et al. (2007). It turns out that several cases can occur, giving different solutions (JeanblancPicqué and Shiryaev overlooked one case that is relevant for their problem). Numerical issues to find solutions are also addressed, and examples are given showing that numerical solutions actually can be found without too much effort.

\section{Results}

We start with a list of assumptions.

Assumption 2.1. We have the following assumptions:

(a) $|\mu(y)|+|\sigma(y)| \leq K(1+y)$ for all $y \geq 0$ and some $K>0$,

(b) $\mu$ and $\sigma$ are continuously differentiable and the derivatives $\mu^{\prime}$ and $\sigma^{\prime}$ are Lipschitz continuous for all $y \geq 0$,

(c) $\sigma^{2}(y)>0$ for all $y \geq 0$,

(d) $\mu^{\prime}(y) \leq r$ for all $y \geq 0$.

The operator $L$ is defined as

$$
L f(y)=\frac{1}{2} \sigma^{2}(y) f^{\prime \prime}(y)+\mu(y) f^{\prime}(y)-r f(y)
$$

for $f \in C^{2}(0, \infty)$, the space of twice continuously differentiable functions on $(0, \infty)$. Note that under Assumption 2.1(b) and (c), any solution to $L f(y)=0$ has $f \in C^{3}(0, \infty)$ and $f^{\prime \prime \prime}(y)$ is Lipschitz continuous; see, e.g. Krylov (1996, Theorem 6.5.3).

Let $g_{1}$ and $g_{2}$ be two independent solutions of $L g=0$, chosen so that $g(y)=g_{1}(0) g_{2}(y)-$ $g_{2}(0) g_{1}(y)$ has $g^{\prime}(0)>0$. Any such solution will be called a canonical solution. Then any solution of $L V(y)=0$ and $V^{\prime}(0)>0$ is of the form

$$
V(y)=\kappa g(y), \quad \kappa>0 .
$$

Remark 2.1. Assumption 2.1(d) may seem a bit unnatural and restrictive. Consider the special case

$$
\mathrm{d} X_{t}=\mu X_{t} \mathrm{~d} t+\sigma\left(X_{t}\right) \mathrm{d} W_{t}, \quad X_{0}=x .
$$

Here, $\mu^{\prime}(x)=\mu$ and also

$$
\mathrm{E}\left[\mathrm{e}^{-r t} X_{t}\right]=x \mathrm{e}^{(\mu-r) t}
$$


If $\mu \leq r$ this stabilizes, but if $\mu>r$ it grows to $\infty$ and therefore it is clearly better to wait. The right quantities to compare are therefore $\mu^{\prime}(x)$ and $r$, one representing the geometric growth rate and the other the geometric discounting rate. The condition $\mu^{\prime}(x) \leq r$ just states that in no state should growth rate exceed discounting rate. In a financial context this is a natural condition, since values are typically calculated under an equivalent martingale measure. In a harvesting context this is a less natural assumption, but dropping it leads to a much more complicated problem, and solutions are then probably best found on a case by case basis.

Before we continue let us briefly recapitulate the general solution when $d_{0}=0$ given by Shreve et al. (1984, Theorem 4.3). According to this result we should look for a solution to $L V(y)=0, y>0$, and a $y^{*}$ that satisfy

$$
V(0)=0, \quad V^{\prime}\left(y^{*}\right)=\frac{1}{1+d_{1}}, \quad \text { and } \quad V^{\prime \prime}\left(y^{*}\right)=0 .
$$

For $y>y^{*}$, set

$$
V(y)=V\left(y^{*}\right)+\frac{y-y^{*}}{1+d_{1}} .
$$

The optimal solution is singular control at barrier $y^{*}$, and $V=V^{*}$ is the value function.

Furthermore, if (2.2) has no solution then there is no optimal control, but the value function is the limit of singular controls at barrier $\bar{y}$ for increasing $\bar{y}$.

We now consider the case in which $d_{0}>0$.

Definition 2.1. A lump sum dividend barrier strategy at $\bar{y}$ and jumpsize $\delta \in(0, \bar{y}]$ satisfies, for $Y_{0-}=y$,

$$
\triangle D_{0}=\frac{y-(\bar{y}-\delta)-d_{0}}{1+d_{1}} \mathbf{1}_{\{y \geq \bar{y}\}}, \quad \Delta D_{t}=\frac{\delta-d_{0}}{1+d_{1}} \mathbf{1}_{\left\{Y_{t-}=\bar{y}\right\}} .
$$

If $\delta<\bar{y}$ it is called a continuation strategy, and if $\delta=\bar{y}$ it is called a ruin strategy. In either case, the corresponding value function is denoted by $V_{\bar{y}, \delta}(y)$. Also, we set $V_{\bar{y}, \delta(\bar{y})}(y)=$ $\sup _{\delta \in(0, \bar{y}]} V_{\bar{y}, \delta}(y)$, so that $\delta(\bar{y})$ is the optimal jumpsize with a lump sum dividend barrier strategy at $\bar{y}$.

A ruin dividend barrier strategy at $\bar{y}$ thus pays out everything in dividends whenever $Y_{t-}$ is equal to or is larger than $\bar{y}$.

The following assumptions are needed for solving the optimality problem for a given barrier.

Assumption 2.2. We have the following assumptions.

(a)

$$
\begin{aligned}
& L V(y)=0, \quad 0<y<\bar{y}, \\
& V(0)=0, \\
& V(y)=V(\bar{y})+\frac{y-\bar{y}}{1+d_{1}}, \quad y>\bar{y},
\end{aligned}
$$

(b)

$$
\begin{aligned}
& V(\bar{y})=V(\bar{y}-\bar{\delta})+\frac{\bar{\delta}-d_{0}}{1+d_{1}}, \quad \bar{\delta} \in(0, \bar{y}), \\
& V^{\prime}(\bar{y}-\bar{\delta})=\frac{1}{1+d_{1}}
\end{aligned}
$$


(c)

$$
V(\bar{y})=\frac{\bar{y}-d_{0}}{1+d_{1}} .
$$

For a lump sum dividend barrier strategy at $\bar{y}$, we may only consider the case where $\bar{y}>d_{0}$ since otherwise costs are higher than income, and this is uninteresting in an optimality context.

The proof of the following result is given in Appendix A.

Proposition 2.1. Assume that Assumption 2.1 holds and that the process $Y$ is a strong solution to (1.1) with $\bar{D}$ as given in (1.3). Assume that $d_{0}>0$ and let $\bar{y} \geq d_{0}$.

(a) If Assumption 2.2(a) and (b) have a solution for unknown $V$ and $\bar{\delta}$, then this solution is unique and $V(y)=V_{\bar{y}, \delta(\bar{y})}(y)$ for all $y \geq 0$ so that the optimal lump sum dividend strategy is a continuation strategy.

(b) If Assumption 2.2(a) and (b) do not have a solution then Assumption 2.2(a) and (c) have a unique solution, $V$. Furthermore, $V(y)=V_{\bar{y}, \bar{y}}(y)$ for all $y \geq 0$, i.e. $\delta(\bar{y})=\bar{y}$, so in this case the optimal lump sum dividend strategy is a ruin strategy.

The first equation in Assumption 2.2(b) (or the equation in Assumption 2.2(c)) just states that the total value before payout should equal the total value after payout plus dividends received, cf. the second part of (1.4). The last equation in Assumption 2.2(a) is actually only a definition. It states that for $y>\bar{y}$, the value $V(y)$ is equal to $V(\bar{y})$ plus the value of the excess $y-\bar{y}$. Note that there is no subtraction of $d_{0}$ here, since in reality the assets will be reduced directly to $\bar{y}-\bar{\delta}$ (or to 0 ), and the fixed cost $d_{0}$, only to be counted once, is included in the first equation of Assumption 2.2(b) (or in Assumption 2.2(c)). Finally, the second equation in Assumption 2.2(b) is just a 'smooth-fit condition'. Its economic interpretation is that the marginal value of receiving one more unit in dividends in excess of $\bar{\delta}$ is equal to the marginal decrease in the value of the company.

In order to present the main result of this paper, we need the following set of assumptions similar to Assumption 2.2.

Assumption 2.3. We have the following assumptions.

(a)

$$
\begin{aligned}
& L V(y)=0, \quad 0<y<y^{*}, \\
& V(0)=0, \\
& V(y)=V\left(y^{*}\right)+\frac{y-y^{*}}{1+d_{1}}, \quad y>y^{*},
\end{aligned}
$$

(b)

$$
\begin{aligned}
& V\left(y^{*}\right)=V\left(y^{*}-\delta^{*}\right)+\frac{\delta^{*}-d_{0}}{1+d_{1}}, \quad \delta^{*} \in\left(0, y^{*}\right), \\
& V^{\prime}\left(y^{*}\right)=\frac{1}{1+d_{1}}, \\
& V^{\prime}\left(y^{*}-\delta^{*}\right)=\frac{1}{1+d_{1}},
\end{aligned}
$$

(c)

$$
V\left(y^{*}\right)=\frac{y^{*}-d_{0}}{1+d_{1}}, \quad V^{\prime}\left(y^{*}\right)=\frac{1}{1+d_{1}} .
$$


Note that Assumption 2.3(a) is only Assumption 2.2(a) with $\bar{y}$ replaced by $y^{*}$, while Assumption 2.3(b) and (c) are Assumption 2.2(b) and (c) with $\bar{y}$ replaced by $y^{*}$ plus the 'smooth-fit condition' $V^{\prime}\left(y^{*}\right)=\left(1+d_{1}\right)^{-1}$. The latter is needed to localize the optimal barrier. The economic interpretation is that at $y^{*}$ the marginal value of keeping money in the company is equal to the marginal value of paying them out as dividends.

It follows, easily from Lemma 2.2(b), below, that if Assumption 2.3(a) and (b) have a solution, then Assumption 2.3(a) and (c) cannot have a solution.

Theorem 2.1. Assume that Assumption 2.1 holds and that the process $Y$ is a strong solution to (1.1) with $\bar{D}$ as given in (1.3) and $d_{0}>0$.

(a) If Assumption 2.3(a) and (b) have a solution for unknown $V, y^{*}$, and $\delta^{*}$, then this solution is unique and $V^{*}(y)=V(y)=V_{y^{*}, \delta^{*}}(y)$ for all $y \geq 0$. Thus, an optimal policy is a lump sum dividend barrier continuation strategy at $y^{*}$ with jumpsize $\delta^{*}$.

(b) If Assumption 2.3(a) and (c) have a solution for unknown $V$ and $y^{*}$, then this solution is unique and $V^{*}(y)=V(y)=V_{y^{*}, y^{*}}(y)$ for all $y \geq 0$. Thus, an optimal policy is a lump sum dividend barrier ruin strategy at $y^{*}$.

(c) In all other cases there does not exist an optimal policy, but

$$
V^{*}(y)=\lim _{\bar{y} \rightarrow \infty} V_{\bar{y}, \delta(\bar{y})}(y)
$$

and this limit exists and is finite for every $y \geq 0$. In terms of a canonical solution,

$$
V^{*}(y)=\frac{1}{1+d_{1}} \frac{1}{\lim _{\bar{y} \rightarrow \infty} g^{\prime}(\bar{y})} g(y) .
$$

The proof of this result is rather lengthy, and most of the technical details are postponed to Appendix A. However, in order to actually solve a concrete problem, it is necessary to gain more insight into the three different cases. In doing so we will also prepare the ground for the full proof. In addition, the investigation below will provide an algorithm for a numerical solution.

We start with the following result, taken from Lemma 4.2 in Shreve et al. (1984).

Lemma 2.1. Let $\mu$ and $\sigma$ satisfy Assumption 2.1(a)-(d) and let $f$ be a solution to $\operatorname{Lf}(y)=0$. Consider the interval $[0, \infty)$.

(a) If $f$ has a 0 on $[0, \infty)$, then $f^{\prime}$ has no 0 on $[0, \infty)$.

(b) If for some $\tilde{y} \in[0, \infty), f^{\prime}(\tilde{y})>0$ and $f^{\prime \prime}(\tilde{y}) \leq 0$, then $f$ is a concave function on $[0, \tilde{y})$.

Lemma 2.2. Let $\mu$ and $\sigma$ satisfy Assumption 2.1(b)-(d) and let $V$ satisfy $L V(y)=0, V(0)=0$, and $V(\hat{y})>0$ for some $\hat{y}>0$.

(a) The function $V$ is strongly increasing.

(b) There exists a $y_{c} \geq 0$ (possibly taking the value $\infty$ ) so that $V$ is concave on $\left(0, y_{c}\right)$ and convex on $\left(y_{c}, \infty\right)$. In particular, $y_{c}=0$ if and only if $\mu(0) \leq 0$ and trivially $V^{\prime \prime}\left(y_{c}\right)=0$ when $0<y_{c}<\infty$. 
Proof. Part (a) follows directly from Lemma 2.1(a). For part (b), note that $\frac{1}{2} \sigma^{2}(0) V^{\prime \prime}(0)=$ $-\mu(0) V^{\prime}(0)$; hence, $\mu(0) \leq 0$ is equivalent to $V^{\prime \prime}(0) \geq 0$ and so $V$ is convex on $[0, \infty)$ by Lemma 2.1(b). If $\mu(0)>0$ then we can define $y_{c}=\sup \left\{y>0: V^{\prime \prime}(y) \leq 0\right\}$. If $y_{c}<\infty$ then, by part (a) and Lemma 2.1(b), $V^{\prime \prime}(y) \leq 0$ for all $y \in\left(0, y_{c}\right]$ and, by the definition of $y_{c}$, $V^{\prime \prime}(y)>0$ for $y \in\left(y_{c}, \infty\right)$. For $y_{c}=\infty$, the proof is given at the beginning of the proof of Theorem 4.2 of Shreve et al. (1984).

Let $g$ be a canonical solution; see (2.1). By Lemma 2.1(a), $g^{\prime}(y)>0$ for all $y$. Let $y_{c}$ be as given in Lemma 2.2(b). If $y_{c}=0$, so that $g$ is convex, or $y_{c}=\infty$, so that it is concave, it is clear that Assumption 2.3(a) and (b) cannot have a solution.

Assume, therefore, that $0<y_{c}<\infty$. In order to have a solution of Assumption 2.3(a) and (b), it is clear that $y^{*}-\delta^{*}<y_{c}<y^{*}$. Let $\kappa_{1}<\kappa_{2}$, and set $V_{i}(y)=\kappa_{i} g(y), i=1,2$. Furthermore, let $y_{i}$ and $\delta_{i}$ satisfy $V_{i}^{\prime}\left(y_{i}-\delta_{i}\right)=V_{i}^{\prime}\left(y_{i}\right)=\left(1+d_{1}\right)^{-1}$ or, equivalently,

$$
g^{\prime}\left(y_{i}-\delta_{i}\right)=g^{\prime}\left(y_{i}\right)=\frac{1}{\kappa_{i}\left(1+d_{1}\right)} \text {. }
$$

From this it follows that

$$
y_{1}-\delta_{1}<y_{2}-\delta_{2}<y_{c}<y_{2}<y_{1} .
$$

Furthermore,

$$
\begin{aligned}
\frac{\delta_{1}}{1+d_{1}}-\left(V_{1}\left(y_{1}\right)-V_{1}\left(y_{1}-\delta_{1}\right)\right) & =\int_{y_{1}-\delta_{1}}^{y_{1}}\left(\left(1+d_{1}\right)^{-1}-\kappa_{1} g^{\prime}(y)\right) \mathrm{d} y \\
& >\int_{y_{2}-\delta_{2}}^{y_{2}}\left(\left(1+d_{1}\right)^{-1}-\kappa_{1} g^{\prime}(y)\right) \mathrm{d} y \\
& >\int_{y_{2}-\delta_{2}}^{y_{2}}\left(\left(1+d_{1}\right)^{-1}-\kappa_{2} g^{\prime}(y)\right) \mathrm{d} y \\
& =\frac{\delta_{2}}{1+d_{1}}-\left(V_{2}\left(y_{2}\right)-V_{2}\left(y_{2}-\delta_{2}\right)\right) .
\end{aligned}
$$

From these considerations, it follows that if there is a solution, this solution is unique. Furthermore, we are provided with a method to find the optimal $\kappa^{*}$ and the corresponding $y^{*}$ and $\delta^{*}$ so that, if possible, Assumption 2.3(a) and (b) are satisfied.

1. Let $g$ be a canonical solution and find $y_{c}$ so that $g^{\prime \prime}\left(y_{c}\right)=0$. If $y_{c}=0$ or $y_{c}=\infty$ then there is no solution. By Lemma 2.2(b), $y_{c}=0$ is equivalent to $\mu(0) \leq 0$, so this case is easy to establish. If $y_{c}=\infty$, then again by Lemma 2.1(b), Theorem 2.1(c) applies.

2. Choose $x_{1}<y_{c}$ and let $\kappa_{1}=1 / g^{\prime}\left(x_{1}\right)\left(1+d_{1}\right)$. Then $V_{1}^{\prime}\left(x_{1}\right)=\kappa_{1} g^{\prime}\left(x_{1}\right)=\left(1+d_{1}\right)^{-1}$.

3. Find (if possible) a $y_{1}>y_{c}$ so that $g^{\prime}\left(y_{1}\right)=1 / \kappa_{1}\left(1+d_{1}\right)$. If this is not possible, try with a larger $x_{1}$ until it is satisfied.

4. Let $\delta_{1}=y_{1}-x_{1}$ and calculate

$$
\frac{\delta_{1}}{1+d_{1}}-\kappa_{1}\left(g\left(y_{1}\right)-g\left(y_{1}-\delta\right)\right) .
$$

If this is larger than $d_{0}\left(1+d_{1}\right)^{-1}$ increase $\kappa_{1}$, i.e. increase $x_{1}$. Otherwise, decrease $x_{1}$. 
5. Repeat the process until a solution is obtained, or until it is clear that there is no solution.

Proposition 2.2. Assume that Assumption 2.1(b)-(d) holds. Then Assumption 2.3(a) and (b) have no solution if and only if either

(i) for all $y, g(y)>g^{\prime}(0)\left(y-d_{0}\right)$,

(ii) for all $y_{0}$ and $y_{1}$ with $y_{0}<y_{1}$, so that

$$
g\left(y_{1}\right)-g\left(y_{0}\right)=g^{\prime}\left(y_{0}\right)\left(y_{1}-y_{0}-d_{0}\right),
$$

it holds that $g^{\prime}\left(y_{0}\right)>g^{\prime}\left(y_{1}\right)$.

Proof. We will first show that part (i) is equivalent to

$$
g\left(y_{1}\right)-g\left(y_{0}\right)>g^{\prime}\left(y_{0}\right)\left(y_{1}-y_{0}-d_{0}\right), \quad 0 \leq y_{0}<y_{1} .
$$

Clearly, (2.3) implies part (i). If $y_{0} \geq y_{c}$ then (2.3) is always satisfied, so assume that $y_{0}<y_{c}$. Then we obtain

$$
\begin{aligned}
\frac{g\left(y_{1}\right)-g\left(y_{0}\right)}{g^{\prime}\left(y_{0}\right)}-\left(y_{1}-y_{0}\right) & =\int_{y_{0}}^{y_{1}}\left(\frac{g^{\prime}(y)}{g^{\prime}\left(y_{0}\right)}-1\right) \mathrm{d} y \\
& \geq \int_{y_{0}}^{y_{1}}\left(\frac{g^{\prime}(y)}{g^{\prime}(0)}-1\right) \mathrm{d} y \\
& \geq \int_{0}^{y_{1}}\left(\frac{g^{\prime}(y)}{g^{\prime}(0)}-1\right) \mathrm{d} y \\
& =\frac{g\left(y_{1}\right)}{g^{\prime}(0)}-y_{1} \\
& >-d_{0}
\end{aligned}
$$

which shows the other implication.

For a solution to Assumption 2.3(a) and (b), $V\left(y^{*}-\delta^{*}\right)=\kappa^{*} g\left(y^{*}-\delta^{*}\right)$, implying that $\kappa^{*}=1 /\left(g^{\prime}\left(y^{*}-\delta^{*}\right)\left(1+d_{1}\right)\right)$; hence, $V\left(y^{*}\right)-V\left(y^{*}-\delta^{*}\right)=\left(\delta^{*}-d_{0}\right) /\left(1+d_{1}\right)$ is equivalent to

$$
g\left(y^{*}\right)-g\left(y^{*}-\delta^{*}\right)=g^{\prime}\left(y^{*}-\delta^{*}\right)\left(\delta^{*}-d_{0}\right),
$$

which is impossible by (2.3). Therefore, there can be no solution.

Assume that part (ii) holds and that a solution exists. The same arguments with $\kappa^{*}$ as above gives

$$
g\left(y^{*}\right)-g\left(y^{*}-\delta^{*}\right)=\frac{\delta^{*}-d_{0}}{\kappa^{*}\left(1+d_{1}\right)} .
$$

But then $1 /\left(\kappa^{*}\left(1+d_{1}\right)\right)=g^{\prime}\left(y^{*}-\delta^{*}\right)>g^{\prime}\left(y^{*}\right)$, a contradiction, and so there cannot be a solution.

It is not hard to be convinced, using the arguments for finding a solution given above, that these are the only cases for which there is no solution.

Remark 2.2. (a) In Theorem 2.1(c), the approximating $V_{\bar{y}, \delta(\bar{y})}$ can (at least in theory) be of both the nonruin type $(\delta(\bar{y})<\bar{y})$ and the ruin type $(\delta(\bar{y})=\bar{y})$. When the canonical solution $g$ is concave, they will be of the nonruin type. The nonruin type is described in Lemma A.5, while the ruin type is described in Lemma A.4. 
(b) If Proposition 2.2(i) applies, then the above search process to find $y^{*}$ and $\delta^{*}$ breaks down at the left-hand tail, i.e. the search will decrease $x_{1}$ to negative values. In this case, either Theorem 2.1(b) or (c) applies. This happens, for example, when $y_{c}=0$, i.e. when $\mu(0) \leq 0$.

(c) When Proposition 2.2(ii) applies, then the search will increase $y_{1}$ to $\infty$, bringing us to Theorem 2.1(c). This happens, for example, when $y_{c}=\infty$, i.e. when $g$ is concave.

(d) Numerically it is Theorem 2.1(c) that is the most difficult to work with since in principle the search should go on to $\infty$, and there is no obvious way to tell when to stop unless this can be done analytically.

(e) If $\lim _{y \rightarrow \infty} g^{\prime}(y)=\infty$, then Theorem 2.1 (a) or (b) apply.

(f) If $g$ is concave then Theorem 2.1(c) applies.

To decide whether an optimal policy exists, i.e. whether Theorem 2.1(a) or (b) applies, the following comparison result may be useful, possibly in conjunction with Remark 2.2(e).

Lemma 2.3. Assume that Assumption 2.1(b) and (c) holds and let $f_{i}(y), i=1,2$, solve

$$
\frac{1}{2} \sigma^{2}(y) f_{i}^{\prime \prime}(y)+\mu_{i}(y) f_{i}^{\prime}(y)-r f_{i}(y)=0, \quad y \geq 0,
$$

where $\mu_{1}(y)>\mu_{2}(y)$ for all $y \geq 0$ and

$$
f_{i}(0)=f_{0} \quad \text { and } \quad f_{i}^{\prime}(0)=f_{1} \geq 0, \quad i=1,2 .
$$

Then $f_{1}^{\prime}(y)<f_{2}^{\prime}(y)$ for all $y>0$, which trivially implies that $f_{1}(y)<f_{2}(y)$ for all $y>0$.

Proof. From $\frac{1}{2} \sigma^{2}(0) f_{i}^{\prime \prime}(0)=r f_{0}-\mu_{i}(0) f_{1}$ it follows that $f_{1}^{\prime \prime}(0)<f_{2}^{\prime \prime}(0)$. By continuity, there exists an $\varepsilon>0$ so that $f_{1}^{\prime \prime}(y)<f_{2}^{\prime \prime}(y)$ for all $y \in[0, \varepsilon]$. Integration from 0 then gives $f_{1}^{\prime}(y)<f_{2}^{\prime}(y)$ and $f_{1}(y)<f_{2}(y)$ for $y \in(0, \varepsilon]$. Let

$$
y_{0}=\inf \left\{y>\varepsilon: f_{1}^{\prime}(y)=f_{2}^{\prime}(y)\right\} .
$$

Assume that $y_{0}<\infty$ and set $f_{i}^{\prime}\left(y_{0}\right)=c$. Clearly, $f_{1}\left(y_{0}\right)<f_{2}\left(y_{0}\right)$ and from

$$
\frac{1}{2} \sigma^{2}\left(y_{0}\right) f_{i}^{\prime \prime}\left(y_{0}\right)=r f_{i}\left(y_{0}\right)-\mu_{i}\left(y_{0}\right) c, \quad i=1,2,
$$

it follows that $f_{1}^{\prime \prime}\left(y_{0}\right)<f_{2}^{\prime \prime}\left(y_{0}\right)$. But, $f_{1}^{\prime \prime}(0)<f_{2}^{\prime \prime}(0)$ as well, and for this to be possible there must exist a $y_{1}<y_{0}$ so that $f_{1}^{\prime}\left(y_{1}\right)=f_{2}^{\prime}\left(y_{1}\right)$, a contradiction. Hence, $y_{0}=\infty$ and the result is proved.

From the general discussion above and the nature of the problem, the following result is rather trivial.

Proposition 2.3. In Theorem 2.1(a) or $(b), y^{*}$ is increasing in $d_{0}$ and, in addition, in Theorem 2.1 (a), $y^{*}-\delta^{*}$ is decreasing in $d_{0}$. In both cases, $y^{*}$ and $y^{*}-\delta^{*}$ are constant with respect to $d_{1}$, while $\kappa^{*}$ is decreasing with respect to both $d_{0}$ and $d_{1}$. Finally, $y^{*}$ is decreasing in $r$. For given $y, V^{*}(y)$ is decreasing in all of $d_{0}, d_{1}$, and $r$; in fact, $V^{*}(y)$ is proportional to $\left(1+d_{1}\right)^{-1}$.

Example 2.1. Let $\mu(y)=\mu$ and $\sigma(y)=\sigma$. Then a canonical solution is

$$
g(y)=\mathrm{e}^{-\theta y} \sinh (\beta y),
$$


TABLE 1: Values of $y^{*}, y^{*}-\delta^{*}$, and a normalized $\kappa^{*}$ for various values of $d_{0}$ when $\sigma^{2}=\mu=1$ and $r=d_{1}=0.1$.

\begin{tabular}{ccccrrrrrr}
\hline$d_{0}$ & 0 & 0.1 & \multicolumn{1}{c}{1} & \multicolumn{1}{c}{5} & \multicolumn{1}{c}{10} & \multicolumn{1}{c}{20} & 22.33 & \multicolumn{1}{c}{30} & \multicolumn{1}{c}{40} \\
\hline$y^{*}$ & 2.82 & 4.11 & 7.02 & 13.71 & 19.85 & 30.46 & 32.82 & 40.48 & 50.47 \\
$y^{*}-\delta^{*}$ & 2.82 & 2.12 & 1.59 & 1.02 & 0.65 & 0.11 & 0.00 & 0.00 & 0.00 \\
$\kappa^{*}$ & 1.00 & 0.92 & 0.70 & 0.37 & 0.21 & 0.07 & 0.06 & 0.03 & 0.01 \\
\hline
\end{tabular}

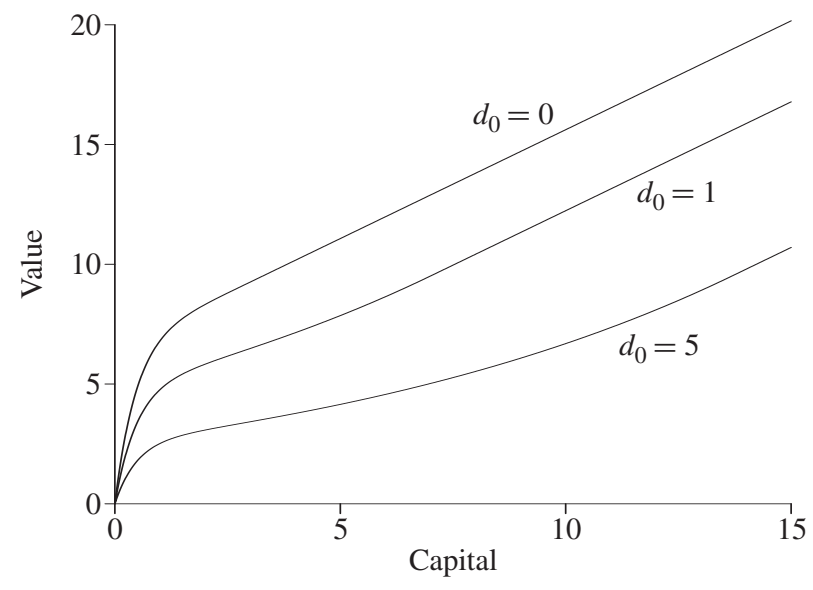

FIGURE 1: The value function $V^{*}(y)$ from Example 2.1 plotted as a function of $y$ for $d_{0}=0, d_{0}=1$, and $d_{0}=5$. The other parameters are the same as in Table 1 .

where

$$
\theta=\frac{\mu}{\sigma^{2}} \quad \text { and } \quad \beta=\frac{1}{\sigma^{2}} \sqrt{2 r \sigma^{2}+\mu^{2}}
$$

This also gives

$$
\begin{aligned}
g^{\prime}(y) & =\mathrm{e}^{-\theta y}(\beta \cosh (\beta y)-\theta \sinh (\beta y)), \\
g^{\prime \prime}(y) & =\mathrm{e}^{-\theta y}\left(\left(\beta^{2}+\theta^{2}\right) \sinh (\beta y)-2 \beta \theta \cosh (\beta y)\right) .
\end{aligned}
$$

Since $\beta>\theta$, it follows, from Remark 2.2(e), that there is an optimal solution.

Table 1 gives values of $y^{*}, y^{*}-\delta^{*}$, and a normalized $\kappa^{*}$ for various values of $d_{0}$ when $\sigma^{2}=\mu=1$ and $r=d_{1}=0.1$. Remember that when $d_{0}=0, y^{*}=y_{c}$. The scaling factor $\kappa^{*}$ was normalized in order to better see the effect of $V^{*}(y)$ for increasing $d_{0}$.

Table 1 was computed in $\mathrm{R}^{\circledR}$ using the search process outlined above. Note the rather large value of $d_{0}$ and corresponding $y^{*}$ before a ruin dividend strategy is better than a nonruin one.

In Figure $1, V^{*}(y)$ is plotted as a function of $y$ for $d_{0}=0, d_{0}=1$, and $d_{0}=5$. The other parameters are the same as in Table 1.

Jeanblanc-Picqué and Shiryaev (1995) studied this particular model with $d_{1}=0$. However, they claimed that Assumption 2.3(a) and (b) always have a solution, which is not correct. From Table 1 we see that when $d_{0}>22.33$, Assumption 2.3(a) and (b) have no solution while Assumption 2.3(a) and (c) have, and the optimal policy is a ruin dividend policy.

Example 2.2. For general $\mu(y)$ and $\sigma(y)$ the Runge-Kutta method was implemented to produce a numerical solution to $\operatorname{Lg}(y)=0$ with $g(0)=0$ and $g^{\prime}(0)=1$. From this $g^{\prime}$ and $g^{\prime \prime}$ were 
calculated and all three were linearly interpolated in order to have functions of all arguments, not only the gridpoints. Then, using these functions, the same search routine as in Example 2.1 was implemented to find $y^{*}, \delta^{*}$, and $\kappa^{*}$. All this was programmed in $\mathrm{R}^{\circledR}$. As a test case, the program was used with the same parameters as in Example 2.1 and with a stepsize 0.01 in the Runge-Kutta solution. The results obtained were for all practical purposes identical with those in Example 2.1. We return to this procedure in Example 3.1, below.

Example 2.3. Let the income process without dividends follow

$$
\mathrm{d} X_{t}=\left(\alpha\left(\rho-X_{t}\right)-\beta\right) \mathrm{d} t+\sigma \mathrm{d} W_{t}, \quad \alpha, \beta, \rho \geq 0 .
$$

This is the mean reverting cash reservoir model suggested by Cadenillas et al. (2007) who conjectured that an optimal solution exists. Here, $\mu(y)=\alpha(\rho-y)-\beta$ and $\mu^{\prime}(y)=-\alpha<r$. Therefore, Assumption 2.1 is satisfied. Let $f_{1}(y)$ and $f_{2}(y)$ satisfy $L_{i} f_{i}(y)=0$ together with $f_{i}(0)=f_{0}$ and $f_{i}^{\prime}(0)=f_{1}>0$, where

$$
L_{i} f_{i}(y)=\frac{1}{2} \sigma^{2} f_{i}^{\prime \prime}(y)+\mu_{i}(y) f_{i}^{\prime}(y)-r f_{i}(y)
$$

with

$$
\mu_{1}(y)=\alpha \rho+\varepsilon \quad \text { and } \quad \mu_{2}(y)=\alpha(\rho-y)-\beta,
$$

for some $\varepsilon>0$. It follows, from Lemma 2.3, that $f_{2}^{\prime}(y)>f_{1}^{\prime}(y)$; hence, an optimal solution exists by Example 2.1 and Remark 2.2(e). This verifies the conjecture of Cadenillas et al. (2007) for the case with a linear cost function. Again, the general method of Example 2.2 can be used to find a numerical solution.

\section{A financial example}

As in Example 2.1, the income process without dividends is assumed to be a linear Brownian motion with drift $\mu$ and diffusion $\sigma$, but now money can be invested in risk free assets with return $r$. However, there are investment costs and they are incurred with rate $\alpha\left(Y_{t}\right)$. The dynamics, (1.1), then become

$$
\mathrm{d} Y_{t}=\left(\mu+\left(r-\alpha\left(Y_{t}\right)\right) Y_{t}\right) \mathrm{d} t+\sigma \mathrm{d} W_{t}-\left(1+d_{1}\right) \mathrm{d} D_{t}-d_{0} \mathbf{1}_{\left\{\triangle D_{t}>0\right\}}, \quad Y_{0-}=y .
$$

For this model, $\mu(y)=\mu+(r-\alpha(y)) y$ and so $\mu^{\prime}(y) \leq r$ for all $y \geq 0$ if and only if

$$
\alpha(y)+\alpha^{\prime}(y) y \geq 0, \quad y \geq 0 .
$$

The total cost of investment intensity is $\alpha\left(Y_{t}\right) Y_{t}$, and a reasonable assumption is that this consists of a fixed part $\alpha_{0}$ and a part $\alpha_{1}$ that is proportional to the amount invested, i.e.

$$
\alpha(y)=\frac{\alpha_{0}}{y}+\alpha_{1}
$$

Clearly, (3.2) is satisfied, and (3.1) becomes

$$
\mathrm{d} Y_{t}=\left(\mu_{0}+\left(r-\alpha_{1}\right) Y_{t}\right) \mathrm{d} t+\sigma \mathrm{d} W_{t}-\left(1+d_{1}\right) \mathrm{d} D_{t}-d_{0} \mathbf{1}_{\left\{\Delta D_{t}>0\right\}}, \quad Y_{0-}=y,
$$

where $\mu_{0}=\mu-\alpha_{0}$. We shall assume that $\mu_{0}>0$ and $0 \leq \alpha_{1}<r$. When $\alpha_{1}=r$, this is Example 2.1 with $\mu_{0}$ replacing $\mu$. 
This problem with $d_{0}=d_{1}=0$ was solved by Cai et al. (2006), but for completeness we start from scratch. Our choice of $g_{1}$ and $g_{2}$ is also slightly different from that of Cai et al.

To find a canonical solution we need to solve

$$
\operatorname{Lg}(y)=\frac{1}{2} \sigma^{2} g^{\prime \prime}(y)+\left(\mu_{0}+\left(r-\alpha_{1}\right) y\right) g^{\prime}(y)-r g(y)=0 .
$$

Substituting $z=-k(y)$ and $f(z)=g(y)$ with

$$
k(y)=\frac{r-\alpha_{1}}{\sigma^{2}}\left(y+\frac{\mu_{0}}{r-\alpha_{1}}\right)^{2},
$$

brings it into the confluent geometric form, i.e.

$$
z f^{\prime \prime}(z)+\left(\frac{1}{2}-z\right) f^{\prime}(z)+\frac{r}{2\left(r-\alpha_{1}\right)} f(z)=0 .
$$

Using the forms $y_{3}$ and $y_{5}$ (see Slater (1960, p. 5)) gives

$$
g(y)=\mathrm{e}^{-k(y)}(U(a, b, k(0)) F(a, b, k(y))-F(a, b, k(0)) U(a, b, k(y))),
$$

where

$$
a=1+\frac{\alpha_{1}}{2\left(r-\alpha_{1}\right)}, \quad b=\frac{1}{2},
$$

and $F$ and $U$ respectively denote the first and second forms of Kummers solution with integral representations

$$
\begin{aligned}
& F(a, b, x)=\frac{\Gamma(b)}{\Gamma(b-a) \Gamma(a)} \int_{0}^{1} \mathrm{e}^{x t} t^{a-1}(1-t)^{b-a-1} \mathrm{~d} t, \quad 0<a<b, \\
& U(a, b, x)=\frac{1}{\Gamma(a)} \int_{0}^{\infty} \mathrm{e}^{-x t} t^{a-1}(1+t)^{b-a-1} \mathrm{~d} t, \quad a>0 .
\end{aligned}
$$

In our case, $0<b<a$; but, using the recursion

$$
F(a, b, x)=\frac{(b+1)(b+x) F(a, b+1, x)-(b+1-a) F(a, b+2, x)}{b(b+1)}
$$

iteratively $m$ times until $b+m>a$, solves the problem. Standard differentiation rules

$$
F^{\prime}(a, b, x)=\frac{a}{b} F(a+1, b+1, x) \quad \text { and } \quad U^{\prime}(a, b, x)=-a U(a+1, b+1, x)
$$

allow us to solve the relevant equations of Assumptions 2.2 and 2.3.

Consider first the case with $\alpha_{1}=0$, i.e. investment costs are constant. Then $a=1$ and furthermore $g_{1}(y)=r y+\mu_{0}$ is a solution, so

$$
\left.g(y)=\mathrm{e}^{-k(0)} U(a, b, k(0))\left(r y+\mu_{0}\right)-\mu_{0} \mathrm{e}^{-k(y)} U(a, b, k(y))\right)
$$

is a canonical solution. Straightforward differentiation gives

$$
\begin{aligned}
& g^{\prime \prime}(y)=\mu_{0} \mathrm{e}^{-k(y)}[\left(k^{\prime \prime}(y)-\left(k^{\prime}(y)\right)^{2}\right)\left(U(a, b, k(y))-U^{\prime}(a, b, k(y))\right) \\
&\left.+\left(k^{\prime}(y)\right)^{2}\left(U^{\prime}(a, b, k(y))-U^{\prime \prime}(a, b, k(y))\right)\right] .
\end{aligned}
$$


Trivially, for the $m$ th derivative,

$$
\operatorname{sgn}\left(U^{(m)}(a, b, k(y))\right)=(-1)^{m},
$$

and for all sufficiently large $y,\left(k^{\prime}(y)\right)^{2}>k^{\prime \prime}(y)$; hence, $g^{\prime \prime}(y)<0$ for all sufficiently large $y$. But it then follows, from Lemma 2.2(a), that $g$ is concave, and therefore no optimal policy exists by Remark 2.2(f). However, by Slater (1960, p. 60), asymptotically as $y \rightarrow \infty$,

$$
\mathrm{e}^{-k(y)} U(a, b, k(y)) \sim \mathrm{e}^{-k(y)}(k(y))^{-a},
$$

and therefore

$$
\lim _{\bar{y} \rightarrow \infty} g^{\prime}(\bar{y})=r \mathrm{e}^{-k(0)} U(a, b, k(0)),
$$

giving

$$
V^{*}(y)=\frac{1}{1+d_{1}}\left(y+\frac{\mu_{0}}{r}\right)-\frac{\mu_{0}}{r} \mathrm{e}^{-(k(y)-k(0))} \frac{1}{\left(1+d_{1}\right) U(a, b, k(0))} U(a, b, k(y)) .
$$

Here the first term is the value if instead of ruin, money could be invested without costs when reaching 0 , and the second term is a reduction in this value due to the possibility of ruin.

Now assume that $0<\alpha_{1}<r$. By Slater (1960, p. 60), asymptotically as $y \rightarrow \infty$,

$$
\mathrm{e}^{-k(y)} F(a, b, k(y)) \sim \frac{\Gamma(b)}{\Gamma(a)}\left(\frac{r-\alpha_{1}}{r}\right)^{r / 2\left(r-\alpha_{1}\right)}\left(y+\frac{\mu_{0}}{r-\alpha_{1}}\right)^{r /\left(r-\alpha_{1}\right)} .
$$

Consequently, by Remark 2.2(e), either Theorem 2.1(a) or (b) apply. Theorem 2.1(b) applies if and only if, for all $y>0$,

$$
\begin{aligned}
& U(a, b, k(0)) F(a, b, k(y))-F(a, b, k(0)) U(a, b, k(y)) \\
& >\left(\frac{a}{b} U(a, b, k(0)) F(a+1, b+1, k(y))+a F(a, b, k(0)) U(a+1, b+1, k(y))\right)\left(y-d_{0}\right) .
\end{aligned}
$$

When $\alpha(y)$ has a more complex structure, there will be no analytical solution to $\operatorname{Lg}(y)=0$, but setting $g(0)=0$ and $g^{\prime}(0)=1$, a numerical solution is readily found. This is outlined in Example 2.2, but before attempting to use this procedure it is useful to know if an optimal strategy exists. To this end, the following result may be helpful.

Proposition 3.1. Assume that Assumption 2.1 holds and let $\alpha(y)$ satisfy (3.2). Furthermore, assume that $\alpha(y) \geq \alpha_{1}$ for all $y \geq 0$, where $\alpha_{1}>0$. Then an optimal dividend strategy exists.

Proof. Let

$$
\mu_{1}(y)=\mu_{1}+\left(r-\alpha_{1}\right) y \quad \text { and } \quad \mu_{2}(y)=\mu+(r-\alpha(y)) y,
$$

where $\mu_{1}$ is chosen large enough so that $\mu_{1}(y)>\mu_{2}(y)$ for all $y \geq 0$. Let $g_{i}$ solve $\operatorname{Lg}_{i}(y)=0$ with $g_{i}(0)=0$ and $g_{i}^{\prime}(0)=1, i=1,2$. By Lemma 2.3, $g_{1}(y)<g_{2}(y)$ for all $y \geq 0$, and by (3.4) asymptotically

$$
g_{1}(y) \sim C\left(y+\frac{\mu_{1}}{r-\alpha_{1}}\right)^{r /\left(r-\alpha_{1}\right)}
$$

for some $C>0$. Therefore, $g_{2}$ is ultimately convex and $\lim _{y \rightarrow \infty} g_{2}^{\prime}(y)=\infty$. Consequently, Theorem 2.2(c) cannot apply by Remark 2.2(e). 
TABLE 2: Values of $y^{*}, y^{*}-\delta^{*}$, and $\kappa^{*}$ for various values of $d_{0}$, when $\sigma^{2}=\mu_{0}=1, r=d_{1}=0.1$, and

\begin{tabular}{cccrrrrrrr}
\hline \multicolumn{10}{c}{$\alpha=0.02}$. \\
\hline$d_{0}$ & 0 & \multicolumn{1}{c}{0.1} & \multicolumn{1}{c}{1} & \multicolumn{1}{c}{5} & \multicolumn{1}{c}{10} & \multicolumn{1}{c}{20} & \multicolumn{1}{c}{30} & \multicolumn{1}{c}{40} & 100 \\
\hline$y^{*}$ & 3.44 & 6.73 & 16.63 & 43.86 & 72.75 & 126.8 & 179.1 & 230.7 & 535.4 \\
$y^{*}-\delta^{*}$ & 3.44 & 2.52 & 2.08 & 1.76 & 1.62 & 1.50 & 1.42 & 1.37 & 1.21 \\
$\kappa^{*}$ & 1.00 & 0.96 & 0.86 & 0.73 & 0.66 & 0.58 & 0.54 & 0.51 & 0.41 \\
\hline
\end{tabular}

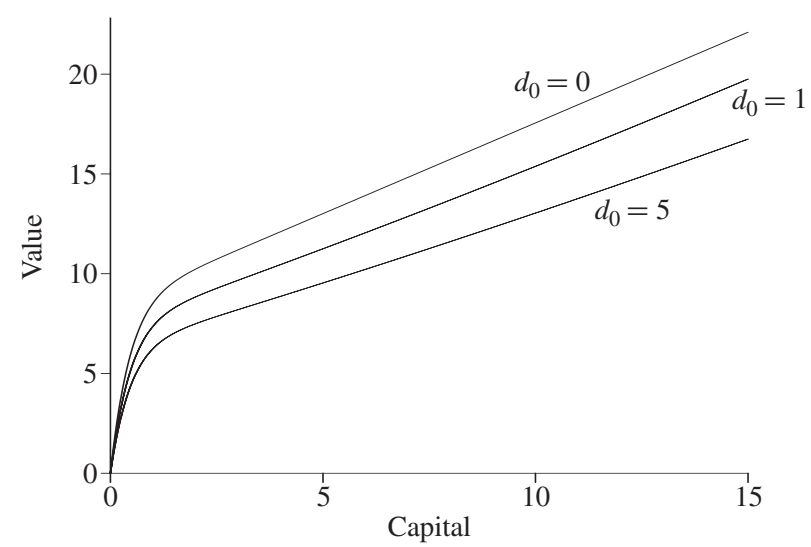

Figure 2: The value function $V^{*}(y)$ from Example 3.1 plotted as a function of $y$ for $d_{0}=0, d_{0}=1$, and $d_{0}=5$. The other parameters are the same as in Table 2 .

Example 3.1. In this example, $Y$ is given by (3.3) with $\mu_{0}=1, \sigma=1, d_{1}=0.1, r=0.1$, and $\alpha=0.02$, i.e. the same as Example 2.1, but now money in the company earns interest at a rate $r$ minus costs incurred at a rate $\alpha$. The program described in Example 2.2 with a stepsize in the Runge-Kutta method equal to 0.01 was used, and the results are given in Table 2. Note the slow decrease in $y^{*}-\delta^{*}$ meaning that the value of $d_{0}$ so that a ruin strategy is preferred is very high. In fact, increasing $d_{0}$ to over 200 led to numerical difficulties and so the critical $d_{0}$ could not be found.

In Figure 2, $V^{*}(y)$ is plotted as a function of $y$ for $d_{0}=0, d_{0}=1$, and $d_{0}=5$. The other parameters are the same as in Table 2. This can be compared with Figure 1.

\section{Appendix A. Proof of Proposition 2.1 and Theorem 2.1}

Throughout the appendix the function $g$ is a canonical solution as defined before (2.1). The point $y_{c}$ is defined in Lemma 2.2(b).

Lemma A.1. Under the conditions of Proposition 2.1(a) or Proposition 2.1(b), we have

$$
V(\bar{y})-V(x) \geq \frac{\bar{y}-x-d_{0}}{1+d_{1}}, \quad 0 \leq x \leq \bar{y} .
$$

Proof. Consider first the case of Proposition 2.1(a), i.e. with $\delta(\bar{y}) \in(0, \bar{y})$, and for simplicity we write $\delta=\delta(\bar{y})$. It is clear that $\bar{y}-\delta<y_{c}$ since otherwise

$$
V(\bar{y})-V(\bar{y}-\delta)>V^{\prime}(\bar{y}-\delta) \delta=\frac{\delta}{1+d_{1}} .
$$


Let

$$
h(x)=V(\bar{y})-V(\bar{y}-x)-\frac{x}{1+d_{1}}, \quad 0 \leq x \leq \bar{y} .
$$

Then

$$
h(0)=0, \quad h(\delta)=-\frac{d_{0}}{1+d_{1}}, \quad h^{\prime}(x)=V^{\prime}(\bar{y}-x)-\frac{1}{1+d_{1}}, \quad h^{\prime \prime}(x)=-V^{\prime \prime}(\bar{y}-x) .
$$

Therefore, $h^{\prime}(\delta)=0$ and $h^{\prime \prime}(\delta)>0$. Furthermore, $h^{\prime}(x)>0$ for $\delta<x \leq \bar{y}$ and, since $h^{\prime}(x)=0$ has at most two roots, it follows that $h$ takes its minimum at $x=\delta$. Uniqueness is obvious and the result follows.

Assume that no such $\delta(\bar{y})$ exists, i.e. the case of Proposition 2.1(b), but, for any given $\delta$, choose $\kappa_{\delta}$ such that

$$
\kappa_{\delta} g^{\prime}(\bar{y}-\delta)=\frac{1}{1+d_{1}} \Longrightarrow \kappa_{\delta}=\frac{1}{g^{\prime}(\bar{y}-\delta)\left(1+d_{1}\right)}
$$

Then, with $V^{\delta}(y)=\kappa_{\delta} g(y), y \leq \bar{y}$,

$$
k(\delta):=\frac{1}{\kappa_{\delta}}\left(V^{\delta}(\bar{y})-V^{\delta}(\bar{y}-\delta)-\frac{\delta-d_{0}}{1+d_{1}}\right)=g(\bar{y})-g(\bar{y}-\delta)-g^{\prime}(\bar{y}-\delta)\left(\delta-d_{0}\right)
$$

has $k(0)=g^{\prime}(\bar{y}) d_{0}>0$. Since $k(\delta)=0$ has no solution, $k(\delta)>0$ for all $\delta$. In particular, with $\delta=\bar{y}$,

$$
\begin{aligned}
g(\bar{y})>g^{\prime}(0)\left(\bar{y}-d_{0}\right) & \Longrightarrow V(\bar{y})=\frac{\bar{y}-d_{0}}{1+d_{1}}>V^{\prime}(0)\left(\bar{y}-d_{0}\right) \\
& \Longrightarrow V^{\prime}(0)<\frac{1}{1+d_{1}} .
\end{aligned}
$$

Let $h(x)$ be given by (A.1) and note that $h(\bar{y})=-d_{0} /\left(1+d_{1}\right)$. By (A.2), $h^{\prime}(\bar{y})<0$ and, furthermore, $h^{\prime}(x)=0$ has at most one root (two roots would imply that $h^{\prime}(\bar{y})>0$ ), and since $h(0)=0, h$ takes its minimum at $x=\bar{y}$ and the result follows.

Proof of Proposition 2.1. Using Lemma A.1, Proposition 2.1 is proved much like, but slightly simpler than, Theorem 2.1(a) and (b), below, and we omit the details.

Lemma A.2. Under the assumptions of Theorem 2.1(a), we have

(a) $L V(y) \leq 0$ for all $y>0$,

(b) $V(y)-V(x) \geq\left(y-x-d_{0}\right) /\left(1+d_{1}\right), 0 \leq x \leq y$,

(c) $y^{*}, \delta^{*}$, and $V$ are uniquely given.

Proof. First note that since $V^{\prime}\left(y^{*}\right)=V^{\prime}\left(y^{*}-\delta^{*}\right)$, by Lemma 2.1(b), $y^{*}-\delta^{*}<y_{c}<y^{*}$. Therefore, $V^{\prime \prime}\left(y^{*}-\right) \geq 0$, and so, by continuity, we have

$$
r V\left(y^{*}\right)=\frac{1}{2} \sigma^{2}\left(y^{*}\right) V^{\prime \prime}\left(y^{*}-\right)+\mu\left(y^{*}\right) V^{\prime}\left(y^{*}\right) \geq \mu\left(y^{*}\right)\left(1+d_{1}\right)^{-1} .
$$


Let $y>y^{*}$, then we obtain

$$
\begin{aligned}
L V(y) & =\frac{\mu(y)}{1+d_{1}}-r\left(V\left(y^{*}\right)+\frac{y-y^{*}}{1+d_{1}}\right) \\
& =\left(1+d_{1}\right)^{-1}\left(\mu(y)-\mu\left(y^{*}\right)-r\left(y-y^{*}\right)\right)+\mu\left(y^{*}\right)\left(1+d_{1}\right)^{-1}-r V\left(y^{*}\right) \\
& \leq\left(1+d_{1}\right)^{-1} \int_{y^{*}}^{y}\left(\mu^{\prime}(x)-r\right) \mathrm{d} x \\
& \leq 0 .
\end{aligned}
$$

To prove part (b), set $\eta^{*}=y^{*}-\delta^{*}$. From the observation at the beginning of the proof, $V^{\prime}(y) \geq\left(1+d_{1}\right)^{-1}$ on $\left(0, \eta^{*}\right)$ and $V^{\prime}(y) \leq\left(1+d_{1}\right)^{-1}$ on $\left(\eta^{*}, \infty\right)$. Therefore,

$$
V(y)-V(x)-\frac{y-x}{1+d_{1}}=\int_{x}^{y}\left(V^{\prime}(u)-\left(1+d_{1}\right)^{-1}\right) \mathrm{d} u
$$

is smallest for $x=\eta^{*}$ and $y \geq y^{*}$, and then it is equal to $-d_{0} /\left(1+d_{1}\right)$. Part (c) is proved in connection with the algorithm preceding Proposition 2.2.

Lemma A.3. Under the assumptions of Theorem 2.1(b), the results of Lemma A.2 hold (with $\left.\delta^{*}=y^{*}\right)$.

Proof. First note that $y^{*}>y_{c}$ since, if $y^{*} \leq y_{c}$ then

$$
V\left(y^{*}\right)=\int_{0}^{y^{*}} V^{\prime}(y) \mathrm{d} y \geq \int_{0}^{y^{*}} V^{\prime}\left(y^{*}\right) \mathrm{d} y=\frac{y^{*}}{1+d_{1}},
$$

which contradicts Assumption 2.3(c). Therefore, part (a) follows as in Lemma A.2(a). By (A.2), with $y^{*}=\bar{y}$,

$$
\frac{y^{*}-d_{0}}{1+d_{1}}=V\left(y^{*}\right)>V^{\prime}(0)\left(y^{*}-d_{0}\right),
$$

and therefore $V^{\prime}(0)<\left(1+d_{1}\right)^{-1}=V^{\prime}\left(y^{*}\right)$. But then $V^{\prime}(y) \leq V^{\prime}\left(y^{*}\right)$ for all $y>0$ $\left(V^{\prime}(y)=V^{\prime}\left(y^{*}\right)\right.$ for $\left.y \geq y^{*}\right)$. This gives, for $0 \leq x \leq y \leq y^{*}$,

$$
\begin{aligned}
V(y)-V(x)-\frac{y-x}{1+d_{1}} & =\int_{x}^{y}\left(V^{\prime}(u)-\left(1+d_{1}\right)^{-1}\right) \mathrm{d} u \\
& \geq \int_{0}^{y^{*}}\left(V^{\prime}(u)-\left(1+d_{1}\right)^{-1}\right) \mathrm{d} u \\
& =V\left(y^{*}\right)-\frac{y^{*}}{1+d_{1}} \\
& =-\frac{d_{0}}{1+d_{1}},
\end{aligned}
$$

which proves part (b) since if $y^{*} \leq x \leq y$, then $V(y)-V(x)=(y-x) /\left(1+d_{1}\right)$.

Again uniqueness follows from the iteration procedure preceding Proposition 2.2.

Lemma A.4. Assume that case (i) of Proposition 2.2 holds and that for any $V$ that satisfies Assumption 2.2(a) the problem

$$
V(\bar{y})=\frac{\bar{y}-d_{0}}{1+d_{1}} \quad \text { and } \quad V^{\prime}(\bar{y})=\frac{1}{1+d_{1}}
$$


has no solution for any $\bar{y}>0$. Then, for $d_{0}<\bar{y}_{1}<\bar{y}_{2}$,

$$
V_{\bar{y}_{1}, \delta\left(\bar{y}_{1}\right)}(y)<V_{\bar{y}_{2}, \delta\left(\bar{y}_{2}\right)}(y), \quad y \geq 0,
$$

and

$$
V(y)=\lim _{\bar{y} \rightarrow \infty} V_{\bar{y}, \delta(\bar{y})}(y)=\kappa g(y)
$$

exists for

$$
\kappa=\frac{1}{1+d_{1}} \lim _{\bar{y} \rightarrow \infty} \frac{1}{g^{\prime}(\bar{y})} .
$$

\section{Furthermore,}

(a) $V(0)=0$,

(b) $L V(y)=0, y>0$,

(c) $V(y)-V(x)>\left(y-x-d_{0}\right) /\left(1+d_{1}\right), 0 \leq x \leq y$.

Proof. For notational simplicity, we write $V_{y}(x)$ for $V_{y, \delta(y)}(x)$ throughout the proof. For $V(\bar{y})=\kappa g(\bar{y})$ to be a solution of (A.3) is equivalent to

$$
g(\bar{y})=g^{\prime}(\bar{y})\left(\bar{y}-d_{0}\right) .
$$

Now, $h(y):=g(y)-g^{\prime}(y)\left(y-d_{0}\right)$ has $h(0)>0$ and, since (A.4) has no solution, we must have

$$
g(y)>g^{\prime}(y)\left(y-d_{0}\right), \quad y>0 .
$$

Let $d_{0}<\bar{y}_{1}<\bar{y}_{2}$ and set $V_{\bar{y}_{i}}(y)=\kappa_{i} g(y)$ for $y \leq y_{i}, i=1,2$. Consider a barrier $\bar{y}>d_{0}$. If Assumption 2.2(a) and (b) have a solution then

$$
g(\bar{y})-g(\bar{y}-\delta(\bar{y}))=g^{\prime}(\bar{y}-\delta(\bar{y}))\left(\delta(\bar{y})-d_{0}\right),
$$

but that contradicts (2.3). Therefore, it is optimal to use a ruin barrier strategy, and so

$$
V_{\bar{y}_{2}}\left(\bar{y}_{1}\right)-V_{\bar{y}_{1}}\left(\bar{y}_{1}\right)=\left(\kappa_{2}-\kappa_{1}\right) g\left(\bar{y}_{1}\right)=\frac{\bar{y}_{2}-d_{0}}{1+d_{1}} \frac{g\left(\bar{y}_{1}\right)}{g\left(\bar{y}_{2}\right)}-\frac{\bar{y}_{1}-d_{0}}{1+d_{1}} .
$$

Proving that this is positive is equivalent to proving that $h\left(\bar{y}_{2}\right)$ is positive, where

$$
h(y)=\frac{g\left(\bar{y}_{1}\right)}{\bar{y}_{1}-d_{0}}-\frac{g(y)}{y-d_{0}} .
$$

But $h\left(\bar{y}_{1}\right)=0$ and

$$
h^{\prime}(y)=\frac{g(y)-g^{\prime}(y)\left(y-d_{0}\right)}{\left(y-d_{0}\right)^{2}},
$$

which is positive by (A.5). Therefore, $\kappa_{1}<\kappa_{2}$, implying that $V_{\bar{y}_{1}}(y)<V_{\bar{y}_{2}}(y)$ for $y \leq \bar{y}_{1}$. Letting $\bar{y}_{1}<y<\bar{y}_{2}$ gives

$$
V_{\bar{y}_{2}}(y)-V_{\bar{y}_{1}}(y)=\frac{\bar{y}_{2}-d_{0}}{1+d_{1}} \frac{g(y)}{g\left(\bar{y}_{2}\right)}-\frac{y-d_{0}}{1+d_{1}},
$$

but this was just proved to be positive. 
Let $\left\{\bar{y}_{n}\right\}$ be increasing to $\infty$ and let $\kappa_{n}$ be given by $V_{\bar{y}_{n}}(y)=\kappa_{n} g(y), y \leq \bar{y}_{n}$, we can then define $\kappa=\lim _{n \rightarrow \infty} \kappa_{n}$. By (A.5), we have

$$
\kappa_{n}=\frac{\bar{y}_{n}-d_{0}}{\left(1+d_{1}\right) g\left(\bar{y}_{n}\right)}<\frac{1}{\left(1+d_{1}\right) g^{\prime}\left(\bar{y}_{n}\right)} .
$$

Since $y_{c}<\infty$, it is necessary that $\lim _{n \rightarrow \infty} g^{\prime}\left(\bar{y}_{n}\right)>0$; hence, $\left\{\kappa_{n}\right\}$ is bounded so $\kappa$ is finite. In fact, since $g$ is ultimately convex, $\kappa$ is as given in Lemma A.4. With $V(y)=\kappa g(y)$, it is clear that $V(0)=0$ and $L V(y)=0$ for all $y$. Furthermore, with $0 \leq x<y$ and $V_{y}(x)=\kappa_{y} g(x)$, we have

$$
V(y)-V(x)=\kappa(g(y)-g(x))>\kappa_{y}(g(y)-g(x))=V_{y}(y)-V_{y}(x) \geq \frac{y-x-d_{0}}{1+d_{1}},
$$

where the last inequality comes from Lemma A.1.

Lemma A.5. Assume that case (ii) of Proposition 2.2 holds. Then the results of Lemma A.4 hold.

Proof. We will first prove that $\lim _{y \rightarrow \infty} g^{\prime}(y) \leq g^{\prime}(0)$. Assume the contrary and let $\left(x_{1}, y_{1}\right)$ satisfy case (ii) of Proposition 2.2. Clearly, $x_{1}<y_{c}$ and therefore $g^{\prime}\left(x_{1}\right) \leq g^{\prime}(0)$, and by assumption $g^{\prime}\left(x_{1}\right) \geq g^{\prime}(y), x_{1}<y<y_{1}$. This gives

$$
-d_{0}=\int_{x_{1}}^{y_{1}}\left(\frac{g^{\prime}(y)}{g^{\prime}\left(x_{1}\right)}-1\right) \mathrm{d} y \geq \int_{0}^{y_{1}}\left(\frac{g^{\prime}(y)}{g^{\prime}(0)}-1\right) \mathrm{d} y .
$$

By assumption, eventually $g^{\prime}(y)>g^{\prime}(0)$, and therefore there exists a $y_{2}>y_{1}$ so that

$$
\int_{0}^{y_{2}}\left(\frac{g^{\prime}(y)}{g^{\prime}(0)}-1\right) \mathrm{d} y=-d_{0} .
$$

But then $g^{\prime}\left(y_{2}\right)>g^{\prime}(0)$, contradicting case (ii) of Proposition 2.2. Hence, $\lim _{y \rightarrow \infty} g(y) \leq$ $g(0)$.

Again, let $\left(x_{1}, y_{1}\right)$ satisfy case (ii) of Proposition 2.2 and let $y_{2}>y_{1}$ be arbitrary. Then, by what we have already proved, we have

$$
-d_{0}=\int_{x_{1}}^{y_{1}}\left(\frac{g^{\prime}(y)}{g^{\prime}\left(x_{1}\right)}-1\right) \mathrm{d} y \geq \int_{0}^{y_{2}}\left(\frac{g^{\prime}(y)}{g^{\prime}(0)}-1\right) \mathrm{d} y .
$$

Increasing the lower limit of integration together with the argument of $g^{\prime}$ in the denominator, we see that there will exist an $x_{2}>0$ so that $g\left(y_{2}\right)-g\left(x_{2}\right)=g^{\prime}\left(x_{2}\right)\left(y_{2}-x_{2}-d_{0}\right)$. Assume that $x_{1} \geq x_{2}$. Then

$$
-d_{0}=\int_{x_{2}}^{y_{2}}\left(\frac{g^{\prime}(y)}{g^{\prime}\left(x_{2}\right)}-1\right) \mathrm{d} y<\int_{x_{1}}^{y_{1}}\left(\frac{g^{\prime}(y)}{g^{\prime}\left(x_{2}\right)}-1\right) \mathrm{d} y<\int_{x_{1}}^{y_{1}}\left(\frac{g^{\prime}(y)}{g^{\prime}\left(x_{1}\right)}-1\right) \mathrm{d} y=-d_{0},
$$

which is a contradiction. To conclude, we have proved that if case (ii) of Proposition 2.2 applies and the pair $\left(x_{1}, y_{1}\right)$ satisfies $g\left(y_{1}\right)-g\left(x_{1}\right)=g^{\prime}\left(x_{1}\right)\left(y_{1}-x_{1}-d_{0}\right)$ then, for any $y_{2}>y_{1}$, there exists an $x_{2}>x_{1}$ so that $\left(x_{2}, y_{2}\right)$ also satisfies this equation.

For $y \leq y_{i}$, let $V_{y_{i}}(y):=V_{y_{i}, \delta\left(y_{i}\right)}(y)=\kappa_{i} g(y), i=1,2$, be the value function of an optimal dividend barrier strategy at $y_{i}$ with jumpsize $\delta_{i}=y_{i}-x_{i}$, where $\left(x_{i}, y_{i}\right)$ are as above. Then,

$$
\kappa_{i}=\frac{1}{g^{\prime}\left(x_{i}\right)\left(1+d_{1}\right)},
$$


and therefore $\kappa_{1}<\kappa_{2}$, which implies that $V_{y_{1}}(y)<V_{y_{2}}(y), y \leq y_{1}$. Next, let $y \in\left(y_{1}, y_{2}\right)$. We will show that $V_{y_{1}}(y)<V_{y_{2}}(y)$ here as well. To do so it is sufficient to show that $V_{y_{1}}\left(y_{2}\right)<V_{y_{2}}\left(y_{2}\right)$ since then $V_{y_{1}}(y)<V_{y}(y)<V_{y_{2}}(y)$. We have

$$
\begin{aligned}
V_{y_{2}}\left(y_{2}\right)-V_{y_{1}}\left(y_{2}\right) & =V_{y_{2}}\left(x_{2}\right)+\frac{y_{2}-x_{2}-d_{0}}{1+d_{1}}-V_{y_{1}}\left(x_{1}\right)-\frac{y_{2}-x_{1}-d_{0}}{1+d_{1}} \\
& =\frac{1}{1+d_{1}}\left(\frac{g\left(x_{2}\right)}{g^{\prime}\left(x_{2}\right)}-\frac{g\left(x_{1}\right)}{g^{\prime}\left(x_{1}\right)}-\left(x_{2}-x_{1}\right)\right) .
\end{aligned}
$$

Let

Then $h\left(x_{1}\right)=0$ and

$$
h(x)=\frac{g(x)}{g^{\prime}(x)}-\frac{g\left(x_{1}\right)}{g^{\prime}\left(x_{1}\right)}-\left(x-x_{1}\right) .
$$

$$
h^{\prime}(x)=-\frac{g(x) g^{\prime \prime}(x)}{\left(g^{\prime}(x)\right)^{2}}>0, \quad x_{1} \leq x \leq x_{2},
$$

since $x_{2}<y_{c}$. This gives the result.

Now assume that $y_{0} \in\left(d_{0}, y_{1}\right)$ is so that $g\left(y_{0}\right)-g(x)=g^{\prime}(x)\left(y_{0}-x-d_{0}\right)$ has no solution for $x \in\left[0, y_{0}\right]$, meaning that an optimal barrier strategy at $y_{0}$ is a ruin strategy. Then $g\left(y_{0}\right)>g^{\prime}(0)\left(y_{0}-d_{0}\right)$ and by Proposition 2.1 with $V_{y_{0}}(y)=\kappa_{0} g(y), 0 \leq y \leq y_{0}$, we have

$$
\begin{aligned}
\left(\kappa_{1}-\kappa_{0}\right) g\left(y_{0}\right) & =V_{y_{1}}\left(y_{0}\right)-V_{y_{0}}\left(y_{0}\right) \\
& =\frac{g\left(y_{0}\right)}{1+d_{1}}\left(\frac{1}{g^{\prime}\left(x_{1}\right)}-\frac{y_{0}-d_{0}}{g\left(y_{0}\right)}\right) \\
& >\frac{g\left(y_{0}\right)}{1+d_{1}}\left(\frac{1}{g^{\prime}\left(x_{1}\right)}-\frac{1}{g^{\prime}(0)}\right) \\
& >0 .
\end{aligned}
$$

Furthermore, in this case

$$
V_{y_{1}}\left(y_{1}\right)-V_{y_{0}}\left(y_{1}\right)=\frac{1}{1+d_{1}}\left(\frac{g\left(x_{1}\right)}{g^{\prime}\left(x_{1}\right)}-x_{1}\right)>0,
$$

since $g$ is concave on $\left[0, x_{1}\right]$. We have therefore proved that when case (ii) of Proposition 2.2 applies with $d_{0}<\bar{y}_{1}<\bar{y}_{2}$, we have

$$
V_{\bar{y}_{1}}(y)<V_{\bar{y}_{2}}(y) .
$$

The rest of the proof is now identical to the last part of the proof of Lemma A.4. The only thing to observe is that for $\left(\bar{x}_{n}, \bar{y}_{n}\right)$ to satisfy the conditions of $\left(x_{i}, y_{i}\right)$ above, it is necessary that $\lim _{n \rightarrow \infty} g^{\prime}\left(\bar{x}_{n}\right)=\lim _{n \rightarrow \infty} g^{\prime}\left(\bar{y}_{n}\right)$, and so $\kappa$ is as given in Lemma A.4.

Proof of Theorem 2.1. Since $V$ is twice continuously differentiable and $D$ has finite variation, the general Itô formula gives

$$
\begin{aligned}
\mathrm{e}^{-r\left(t \wedge \tau_{y}\right)} V\left(Y_{t \wedge \tau_{y}}\right)= & V(y)+\int_{0}^{t \wedge \tau_{y}} \mathrm{e}^{-r s} L V\left(Y_{s}\right) \mathrm{d} s+\int_{0}^{t \wedge \tau_{y}} \mathrm{e}^{-r s} \sigma\left(Y_{s}\right) V^{\prime}\left(Y_{s}\right) \mathrm{d} W_{s} \\
& -\left(1+d_{1}\right) \int_{0}^{t \wedge \tau_{y}} \mathrm{e}^{-r s} V^{\prime}\left(Y_{s}\right) \mathrm{d} D_{s}^{\mathrm{c}}-\sum_{s \leq t \wedge \tau_{y}} \mathrm{e}^{-r s}\left(V\left(Y_{s-}\right)-V\left(Y_{s}\right)\right),
\end{aligned}
$$


where $D^{\mathrm{c}}$ denotes the continuous part of $D$. But since there is a fixed cost with each payment, $D^{\mathrm{c}} \equiv 0$. Letting $\rho_{n}=\inf \left\{t: Y_{t} \geq n\right\}$, by (A.6) and Lemmas A.2-A.5, we obtain

$$
\begin{aligned}
\mathrm{e}^{-r\left(t \wedge \tau_{y} \wedge \rho_{n}\right)} V\left(Y_{t \wedge \tau_{y} \wedge \rho_{n}}\right) \leq & V(y)+\int_{0}^{t \wedge \tau_{y} \wedge \rho_{n}} \mathrm{e}^{-r s} \sigma\left(Y_{s}\right) V^{\prime}\left(Y_{s}\right) \mathrm{d} W_{s} \\
& -\sum_{s \leq t \wedge \tau_{y} \wedge \rho_{n}} \mathrm{e}^{-r s}\left(V\left(Y_{s-}\right)-V\left(Y_{s-}+\Delta Y_{s}\right)\right) \\
\leq & V(y)+\int_{0}^{t \wedge \tau_{y} \wedge \rho_{n}} \mathrm{e}^{-r s} \sigma\left(Y_{s}\right) V^{\prime}\left(Y_{s}\right) \mathrm{d} W_{s} \\
& -\sum_{s \leq t \wedge \tau_{y} \wedge \rho_{n}} \mathrm{e}^{-r s} \frac{\left(-\triangle Y_{s}\right)-d_{0}}{1+d_{1}} \\
= & V(y)+\int_{0}^{t \wedge \tau_{y} \wedge \rho_{n}} \mathrm{e}^{-r s} \sigma\left(Y_{s}\right) V^{\prime}\left(Y_{s}\right) \mathrm{d} W_{s}-\sum_{s \leq t \wedge \tau_{y} \wedge \rho_{n}} \mathrm{e}^{-r s} \Delta D_{s} .
\end{aligned}
$$

Taking expectations gives

$V(y) \geq \mathrm{E}^{y}\left[\int_{0}^{t \wedge \tau_{y} \wedge \rho_{n}} \mathrm{e}^{-r s} \mathrm{~d} D_{s}\right]+\mathrm{E}^{y}\left[\mathrm{e}^{-r\left(t \wedge \tau_{y} \wedge \rho_{n}\right)} V\left(Y_{t \wedge \tau_{y} \wedge \rho_{n}}\right)\right] \geq \mathrm{E}^{y}\left[\int_{0}^{t \wedge \tau_{y} \wedge \rho_{n}} \mathrm{e}^{-r s} \mathrm{~d} D_{s}\right]$.

Finally, letting $t \rightarrow \infty$ and $n \rightarrow \infty$ gives, by monotone convergence,

$$
V(y) \geq \mathrm{E}^{y}\left[\int_{0}^{\tau_{y}} \mathrm{e}^{-r s} \mathrm{~d} D_{s}\right]
$$

For the strategies given in Theorem 2.1(a) and (b), $Y$ and $V^{\prime}$ are bounded and $L V\left(Y_{S}\right)=0$ for all $s$ since $Y_{s} \leq y^{*}$. Therefore, by Lemmas A.2 and A.3, we have

$$
V(y)=\mathrm{E}^{y}\left[\int_{0}^{t \wedge \tau_{y}} \mathrm{e}^{-r s} \mathrm{~d} D_{s}\right]+\mathrm{E}^{y}\left[\mathrm{e}^{-r\left(t \wedge \tau_{y}\right)} V\left(Y_{t \wedge \tau_{y}}\right)\right] .
$$

Letting $t \rightarrow \infty$ and using monotone convergence on the first term on the right-hand side and dominated convergence on the second term on the right-hand side together with $V(0)=0$ gives the result for parts (a) and (b).

Now assume that the assumptions of Theorem 2.1(a) and (b) do not hold, and let $D^{*}$ be an optimal strategy. Let $D^{\prime}$ be 'the more generous payout'

$$
\Delta D_{s}^{\prime}=V\left(Y_{s-}\right)-V\left(Y_{s-}+\Delta Y_{s}\right)>\Delta D_{s}^{*} \text { when } \Delta D_{s}^{*}>0,
$$

where the inequality follows from Lemmas A.4 and A.5. Therefore,

$$
\mathrm{P}\left(D_{t \wedge \tau_{y}}^{\prime}>D_{t \wedge \tau_{y}}^{*}\right)>0
$$

for sufficiently large $t$ (if not $\mathrm{E}^{y}\left[\int_{0-}^{\tau_{y}} \mathrm{e}^{-r s} \mathrm{~d} D_{s}^{*}\right]=0$, and $D^{*}$ is not optimal). Consequently, as in the first part of the proof, we have

$$
V(y) \geq \mathrm{E}^{y}\left[\int_{0-}^{\tau_{y}} \mathrm{e}^{-r s} \mathrm{~d} D_{s}^{\prime}\right]>\mathrm{E}^{y}\left[\int_{0-}^{\tau_{y}} \mathrm{e}^{-r s} \mathrm{~d} D_{s}^{*}\right] .
$$


Let

$$
\varepsilon=V(y)-\mathrm{E}^{y}\left[\int_{0-}^{\tau_{y}} \mathrm{e}^{-r s} \mathrm{~d} D_{s}^{*}\right] .
$$

By Lemmas A.4 and A.5, there exists a $\bar{y}$ so that $V(y)-V_{\bar{y}, \delta(\bar{y})}(y)<\varepsilon$, and so $D^{*}$ cannot be optimal. It follows, from Lemmas A.4 and A.5, that $V(y)=\lim _{\bar{y} \rightarrow \infty} V_{\bar{y}, \delta(\bar{y})}(y)$. This completes the proof.

\section{References}

Alvarez, L. H. R. (2004a). A class of solvable impulse control problems. Appl. Math. Optimization 49, 265-295.

Alvarez, L. H. R. (2004b). Stochastic forest stand value and optimal timber harvesting. SIAM J. Control Optimization 42, 1972-1993.

Asmussen, S. and TAKsar, M. (1997). Controlled diffusion models for optimal dividend pay-out. Insurance Math. Econom. 20, 1-15.

Cadenillas, A., Sarkar, S. and Zapatero, F. (2007). Optimal dividend policy with mean-reverting cash reservoir. Math. Finance 17, 81-109.

Cadenillas, A., Choulli, T., Taksar, M. and Zhang, L. (2006). Classical and impulse stochastic control for the optimization of the dividend and risk policies of an insurance firm. Math. Finance 16, 181-202.

CaI, J., Gerber, H. U. and Yang, H. (2006). Optimal dividends in an Ornstein-Uhlenbeck type model with credit and debit interest. N. Amer. Actuarial J. 10,94-119.

Choulli, T., Taksar, M. and Zhou, X. Y. (2003). A diffusion model for optimal dividend distribution for a company with constraints on risk control. SIAM J. Control Optimization 46, 1946-1979.

DÉcamps, J. P. And Villeneuve, S. (2007). Optimal dividend policy and growth option. Finance Stoch. 11, 3-27.

Gerber, H. U. ANd ShiU, E. S. W. (2004). Optimal dividends: analysis with Brownian motion. N. Amer. Actuarial J. 8, 1-20.

Grandits, P., Hubalek, F., Schachermayer, W. and Zigo, M. (2007). Optimal expected exponential utility of dividend payments in a Brownian risk model. Scand. Actuarial J., 73-107

HøJGAARD, B. AND TAKSAR, M. (2004). Optimal dynamic portfolio selection for a corporation with controllable risk and dividend distribution policy. Quantitative Finance 4, 315-327.

Jeanblanc-Picqué, M. and Shiryaev, A. N. (1995). Optimization of the flow of dividends. Russian Math. Surveys 50, 257-277.

Krylov, N. V. (1996). Lectures on Elliptic and Parabolic Equations in Hölder Spaces (Graduate Studies Math. 12). American Mathematical Society, Providence, RI.

LunGU, E. M. AND ØKSENDAL, B. (1997). Optimal harvesting from a population in a stochastic crowded environment. Math. Biosci. 145, 47-75.

PAulsen, J. (2003). Optimal dividend payouts for diffusions with solvency constraints. Finance Stoch. 7, 457-473.

Paulsen, J. And Gjessing, H. K. (1997). Optimal choice of dividend barriers for a risk process with stochastic return on investments. Insurance Math. Econom. 20, 215-223.

Shreve, S. E., LehoczKy, J. P. AND Gaver, D. P. (1984). Optimal consumption for general diffusions with absorbing and reflecting barriers. SIAM J. Control Optimization 22, 55-75.

Slater, L. J. (1960). Confluent Hypergeometric Functions. Cambridge University Press. 